\title{
Intervenção educativa na qualidade de vida e conhecimento da síndrome metabólica
}

Educational intervention in quality of life and knowledge of metabolic syndrome Intervención educativa en la calidad de vida y conocimientos del síndrome metabólico

Isleide Santana Cardoso Santos ${ }^{1}$ (i) https://orcid.org/0000-0001-8671-8686

Wilkslam Alves de Araújo ${ }^{1}$ io https://orcid.org/0000-0002-3323-4650

Taynnan de Oliveira Damaceno ${ }^{1}$ (6) https://orcid.org/0000-0002-7507-7593

Andréa dos Santos Souza ${ }^{2}$ ic https://orcid.org/0000-0001-8269-6029

Rita Narriman Silva de Oliveira Boery ${ }^{1}$ ic nttps://orcid.org/0000-0002-7823-9498

Josicélia Dumêt Fernandes ${ }^{3}$ iD https://orcid.org/0000-0003-2946-5314

Como citar:

Santos IS, Araújo WA, Damaceno TO, Souza AS, Boery RN, Fernandes JD. Intervenção educativa na qualidade de vida e conhecimento da síndrome metabólica. Acta Paul Enferm. 2022;35:eAPE02982.

DOI

http://dx.doi.org/10.37689/acta-ape/2022A002982

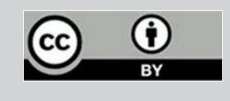

Descritores

Enfermagem em saúde comunitária; Educação em saúde; Educação de pacientes; Síndrome metabólica; Qualidade de

vida; Fatores de risco

Keywords

Community health nursing: Health education; Patien education; Metabolic syndrome; Quality of life; Risk factors

Descriptores

Enfermería en salud comunitária; Educación en salud Educación del paciente; Síndrome metabólico; Calidad de vida; Factores de riesgo

Submetido 9 de Outubro de 2020

Aceito

26 de Maio de 2021

Autor correspondente Isleide Santana Cardoso Santos E-mail: isleide71@yahoo.com.br

Editor Associado (Avaliação pelos pares): Camila Takao Lopes (https://orcid.org/0000-0002-6243-6497) Escola Paulista de Enfermagem, Universidade Federal de São Paulo, São Paulo, SP, Brasil

\section{Resumo}

Objetivo: Avaliar a efetividade de um programa educativo de promoção à saúde na melhora dos domínios da qualidade de vida e no conhecimento da síndrome metabólica.

Métodos: Ensaio clínico não-randomizado incluindo 61 adultos com síndrome metabólica (49 $\pm 7,6$ anos). Os participantes foram intencionalmente alocados em dois grupos: intervenção $(n=31)$ e controle $(n=30) .0$ desfecho primário foi a mudança do conhecimento sobre síndrome metabólica e seus fatores de risco, e 0 secundário, a melhora da qualidade de vida (SF-36) avaliados em dois momentos, na condição basal e ao final da intervenção (seis meses). Foram utilizados os testes U de Mann Whitney e Wilcoxon, adotando-se nível de significância de $5 \%$.

Resultados: 0 programa educativo melhorou 0 domínio de dor corporal da qualidade de vida no grupo intervenção ( $p=0,01)$, embora sem diferença estatística entre os grupos. 0 conhecimento sobre hipertensão e diabetes $(p=0,02)$, síndrome metabólica $(p<0,001)$ e conhecimento geral $(p<0,001)$ apresentaram aumento significativo no grupo intervenção aos seis meses, o que não ocorreu no grupo controle.

Conclusão: 0 programa educativo pode ser uma estratégia efetiva para melhorar a qualidade de vida com destaque para o domínio de dor corporal e aumentar o conhecimento da síndrome metabólica em adultos com a síndrome.

\section{Abstract}

Objective: To assess the effectiveness of an educational health promotion program in improving quality of life and knowledge domains of metabolic syndrome.

Methods: This is a non-randomized clinical trial including 61 adults with metabolic syndrome (49 \pm 7.6 years). Participants were intentionally divided into two groups: intervention $(n=31)$ and control $(n=30)$. The primary outcome was the change in knowledge about metabolic syndrome and its risk factors, and the secondary, the improvement in quality of life (SF-36) assessed in two moments, at baseline and at the end of intervention (six months). Mann Whitney and Wilcoxon U tests were used, adopting a significance level of $5 \%$.

Results: The educational program improved the body ache domain of quality of life in the intervention group $(p=0.01)$, although with no statistical difference between the groups. Knowledge about hypertension and diabetes $(p=0.02)$, metabolic syndrome $(p<0.001)$ and general knowledge $(p<0.001)$ showed a significant increase in the intervention group at six months, which did not occur in the control group.

Conclusion: The educational program can be an effective strategy to improve quality of life, highlighting the domain of body ache and increasing knowledge of metabolic syndrome in adults with the syndrome.

Universidade Estadual do Sudoeste da Bahia, Vitória da Conquista, BA, Brasil.

2Universidade Estadual de Santa Cruz, llhéus, BA, Brasil.

UUniversidade Federal da Bahia, Salvador, BA, Brasil.

Conflitos de interesse: nada a declarar. 


\section{Resumen}

Objetivo: Evaluar la efectividad de un programa educativo de promoción de la salud en la mejora de los dominios de calidad de vida y en los conocimientos del síndrome metabólico.

Métodos: Ensayo clínico no aleatorizado, que incluyó 61 adultos con síndrome metabólico (49 7,6 años). Los participantes fueron intencionalmente separados en dos grupos: experimental $(n=31)$ y de control $(n=30)$. El criterio principal de valoración fue el cambio de conocimientos sobre síndrome metabólico y sus factores de riesgo, y el secundario, la mejora de la calidad de vida (SF-36), evaluados en dos momentos: en condición basal y al final de la intervención (seis meses). Se utilizaron las pruebas U de Mann-Whitney y de Wilcoxon y se adoptó un nivel de significación de $5 \%$.

Resultados: El programa educativo mejoró el dominio de dolor corporal de la calidad de vida en el grupo experimental ( $p=0,01)$, aunque no hubo diferencia estadística entre los grupos. Los conocimientos sobre hipertensión y diabetes $(p=0,02)$, síndrome metabólico $(p<0,001)$ y conocimientos generales $(p<0,001)$ presentaron aumento significativo en el grupo experimental a los seis meses, lo que no ocurrió en el grupo de control.

Conclusión: El programa educativo puede ser una estrategia efectiva para mejorar la calidad de vida, con énfasis en el dominio de dolor corporal, y aumentar los conocimientos sobre el síndrome metabólico en adultos con el síndrome.

Registro de Ensaio Clínico Brasileiro (REBEC): RBR-43K52N

\section{Introdução}

A Síndrome Metabólica (SM) é desencadeada pela agregação de marcadores de risco cardiovascular que envolve etiologia multifatorial. O diagnóstico da SM é baseado em um indivíduo com pelo menos três dos seguintes fatores de risco: aumento da circunferência abdominal, elevaçấo da pressão arterial, da glicemia vascular de jejum, dos triglicerídeos el ou baixos níveis de lipoproteína-colesterol de alta densidade (HDL-c)..$^{(1,2)}$

Além dos fatores de risco que definem a SM outros fatores podem contribuir para desencadear a síndrome, como por exemplo a manutenção de um estilo de vida não saudável e falta de conhecimento. Sabe-se também que pessoas com SM apresentam consequentemente comprometimento da qualidade de vida e têm risco aumentado de morbimortalidade relacionadas a doenças cardiovasculares e diabetes mellitus tipo $2 .^{(1,3)}$ Os dados de um estudo transversal, de base populacional, constatou prevalência de $38,4 \%$ da SM na populaçáo adulta brasileira, mostrou também que quanto menor o nível de escolaridade, maior as prevalências dos componentes da SM. ${ }^{(4)}$

A modificação do estilo de vida tem sido recomendada como medida terapêutica para indivíduos com SM. Trata-se de uma abordagem abrangente baseada em exercícios, alimentação, educação em saúde e farmacoterapia. ${ }^{(5)}$ Estudos relataram efeitos significativos de programas para modificaçáo do estilo de vida nos domínios da qualidade de vida de pessoas com SM..$^{(5,7)}$ No entanto, a maioria das intervenções abordam especialmente exercícios e dieta de maneira isolada, in- tensiva e/ou restritiva. ${ }^{(8,9)}$ Além disso, pouco se sabe sobre os efeitos no incremento do conhecimento em relaçáo aos parâmetros metabólicos e cardiovasculares no contexto da SM, visto que os resultados são principalmente demonstrados nos marcadores fisiológicos da SM. ${ }^{(5,10)}$ Portanto, desenvolveu-se um programa educativo multidisciplinar de promoçâo da saúde, liderado por enfermeiros, com incentivo para mudanças do estilo de vida das pessoas com SM por meio da compreensão das relaçôes de causas-efeitos.

Este estudo teve como objetivo avaliar a efetividade de um programa educativo de promoção à saúde na melhora dos domínios da qualidade de vida e no conhecimento da SM.

\section{Métodos}

Ensaio clínico não-randomizado, controlado, aberto, com dois braços. Foi realizado em um centro de saúde da atenção primária, na zona urbana do município de Jequié, BA, Brasil.

Foram incluídos no estudo homens e mulheres entre 18 e 59 anos de idade, com três ou mais componentes clínicos para SM, conforme critérios do Third Report of the National Cholesterol Education Program Expert Panelon Detection, Evaluation and Treatment of High Blood Cholesterol in Adults

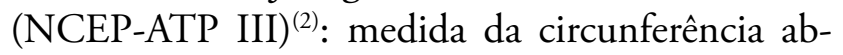
dominal $>88 \mathrm{~cm}$ para mulheres e $>102 \mathrm{~cm}$ para homens; pressão arterial sistólica $\geq 130 \mathrm{mmHg}$, pressão arterial diastólica $\geq 85 \mathrm{mmHg}$; glicemia de jejum $\geq 100 \mathrm{mg} / \mathrm{dl}$; triglicerídeos $\geq 150 \mathrm{mg} / \mathrm{dl}$; HDL-c $<40$ 
$\mathrm{mg} / \mathrm{dl}$ para homens e $<50 \mathrm{mg} / \mathrm{dl}$ para mulheres; e/ ou glicemia de jejum $\geq 100 \mathrm{mg} / \mathrm{dl}$. Foram excluídos indivíduos que apresentaram alguma das seguintes situaçôes: adultos com diagnóstico de diabetes tipo I; gestantes; comprometimento cognitivo; e apresentar número superior a $50 \%$ de faltas nas oficinas.

O tamanho amostral foi de 80 voluntários, considerando-se um effect size de 0.25 , erro alfa de $5 \%$, um poder estatístico de $80 \%$ e perda amostral de $20 \%$. O recrutamento seguiu protocolo de triagem padronizado pela equipe de pesquisadores, sendo convidados a participar do estudo os indivíduos que compareceram para atendimento habitual no centro de saúde e atenderam aos critérios de elegibilidade.

Entre os voluntários $(\mathrm{n}=108), 80$ indivíduos do centro de saúde foram considerados elegíveis $(74,1 \%)$. Após triagem inicial, os participantes foram intencionalmente alocados em dois grupos de 40 indivíduos: intervenção (Cuidar Educando na Síndrome Metabólica, G-CESM), que participou do programa educativo de promoção da saúde, e controle (G-controle), que manteve atendimento habitual. No G-CESM, nove participantes foram excluídos: uma por ter engravidado e oito por apresentarem baixa frequência às oficinas. No G-controle, dez participantes foram excluídos: dois mudaram-se para outra cidade e oito náo tiveram interesse de continuar no estudo. O Fluxograma de distribuição dos participantes do estudo é apresentado na figura 1 .

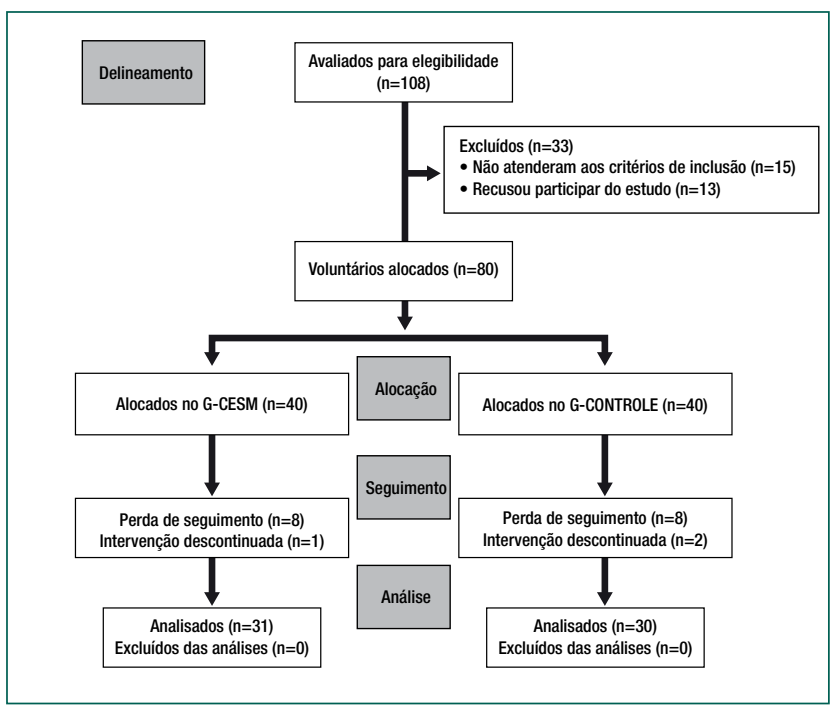

Figura 1. Fluxograma de distribuição dos participantes
O G-CESM participou do programa de promoção da saúde que consistiu de sete oficinas, mensais, em grupo, planejadas e fundamentadas na Pedagogia da Autonomia ${ }^{(1)}$ para promoção da saúde, com possibilidade de mudanças no conhecimento e no estilo de vida. O conteúdo do programa foi desenvolvido com base nas diretrizes clínicas para pessoas com $S^{(12-14)}$ e em cooperação com os interesses do grupo. Cada uma com duração de 90 a 120 minutos, lideradas por enfermeiros e estruturada em dois momentos, no primeiro os enfermeiros do projeto abordavam o conteúdo programado e no segundo momento um profissional de saúde convidado conversava com os participantes sobre assuntos de interesse do grupo, que eram definidos no final de cada oficina. Portanto, os participantes eram recepcionados, e em seguida iniciava-se a abordagem do conteúdo programando abrangendo a SM e seus fatores de risco (conceito, diagnóstico, tratamento e complicações), alimentação saudável, comportamento sedentário, dores, estresse e ansiedade, ergonomia, atividades integrativas, distúrbios metabólicos e cardiovasculares. Os profissionais convidados foram os seguintes: educador físico, enfermeiro, fisioterapeuta, farmacêutico, médico cardiologista, nutricionista e psicólogo. O programa foi conduzido e realizado por três enfermeiros do Grupo de Pesquisa Saúde e Qualidade de Vida (UESB), que estabeleceram relaçôes cordiais com os participantes do grupo e receberam as mesmas instruções e treinamento para colaborar na execução da intervenção.

O G-controle não participou do programa educativo e, assim como o G-CESM, manteve o atendimento habitual no centro de saúde, com consulta mensal. Eles receberam mensalmente uma ligação telefônica para confirmar a participação no estudo, comparecendo no centro de saúde para cumprir o agendamento. Além das mediçôes programadas, não houve outro tipo de contato pessoal dos pesquisadores com o grupo controle durante o estudo.

Os grupos foram avaliados na condição basal (pré-intervenção) e seis meses após do período de intervenção (pós-intervenção). Os encontros para coleta de dados foram previamente agendados por telefone e realizados por uma equipe de pesquisa- 
dores treinada, pela pesquisadora principal, para garantir a qualidade da produção de dados, sem vieses. As avaliaçóes dos participantes foram realizadas separadamente em sala privativa no centro de saúde, para evitar que as respostas de uns influenciassem a dos outros.

Os dados sociodemográficos foram coletados na condição basal por meio de entrevistas individuais, utilizando-se um questionário estruturado em três campos gerais, a saber: identificação pessoal (sexo, idade, cor, situação conjugal, escolaridade e renda); aspectos gerais de saúde (diabetes e hipertensão) e estilo de vida (hábito tabágico, uso de álcool e prática de atividade física).

As variáveis antropométricas de peso $(\mathrm{kg})$ e estatura $(\mathrm{cm})$ foram medidas nos momentos pré-intervenção e pós-intervenção, uma única vez por um mesmo pesquisador. $\mathrm{O}$ peso foi obtido com os participantes descalços e com roupas leves, em uma balança digital portátil (Wiso ${ }^{\oplus}$, modelo W801) com capacidade de $0-180 \mathrm{~kg}$ e precisão de $100 \mathrm{~g}$. A estatura foi avaliada com um estadiômetro metálico portátil (Sanny, modelo capriche), com resolução de $0,1 \mathrm{~mm}$. Após a mensuração das duas medidas, realizou-se o cálculo do o índice de massa corporal $\left(\mathrm{IMC}=\right.$ peso $(\mathrm{kg}) /$ altura $\mathrm{x}$ altura $\left.\left(\mathrm{m}^{2}\right)\right)$. Foram considerados eutróficos os indivíduos com valores de IMC situados entre 18,5 a $24,9 \mathrm{Kg} / \mathrm{m}^{2}$, com sobrepeso entre 25,0 e $29,9 \mathrm{~kg} / \mathrm{m}^{2}$; e com obesidade, àqueles com $\mathrm{IMC} \geq 30 \mathrm{~kg} / \mathrm{m}^{2}$. ${ }^{(15)}$

A qualidade de vida foi avaliada nos momentos pré-intervenção e pós-intervenção usando o Questionário de Qualidade de Vida SF-36, que consiste em um questionário formado por 36 itens divididos em oito domínios: capacidade funcional; limitação por aspectos físicos; dor corporal; estado geral de saúde; vitalidade; aspectos sociais; limitação por aspectos emocionais; e saúde mental. $\mathrm{O}$ escore final varia de 0 a 100 para cada domínio (obtido por meio de cálculo do Raw Scale), os mais altos escores indica melhor qualidade de vida. ${ }^{(16)}$

A avaliação do conhecimento sobre SM e dos seus fatores de riscos, foi verificada nos momentos pré-intervenção e pós-intervenção por meio de um questionário estruturado, elaborado para o estudo, composto por 36 perguntas distribuídas em oito grupos temáticos: diabetes e hipertensão (7 itens); tabagismo e álcool (3 itens); estresse (3 itens); sedentarismo (3 itens); medicamentos (2 itens); alimentação (9 itens); Síndrome Metabólica ( 9 itens) e conhecimento geral (a soma de todos os itens). Para cada pergunta respondida corretamente foi atribuído o valor de um ponto, somando um total máximo de 36 pontos de conhecimento geral. As comparaçóes para verificar o incremento no conhecimento foram feitas entre os grupos e ao longo do tempo.

O tratamento estatístico foi realizado através do Statistical Package for the Social Sciences (SPSS, versão 21.0). Os dados categóricos foram descritos em frequência relativa e absoluta, enquanto as contínuas foram expressas como mediana e intervalo interquartil (IQ). A ausência de normalidade dos dados foi verificada pelo teste de Kolmogorov-Smirnov e análise da homogeneidade das variâncias foi realizada pelo teste de Levene. Para tanto, utilizou-se o teste U de Mann Whitney para comparaçáo dos dados contínuos entre os grupos (G-CESM e G-controle), e aplicou-se o teste de Wilcoxon para análise intragrupos nos momentos pré e pós-intervenção. Para todas as análises foi considerado o nível de significância de 5\%.

O projeto foi submetido e aprovado pelo Comitê de Ética em Pesquisa da Universidade Estadual do Sudoeste da Bahia (UESB, número CAAE 92352818.9.0000.0055, parecer: 2.850.239), e registrado no Registro de Ensaio Clínico Brasileiro (REBEC, número RBR-43K52N).

\section{Resultados}

Foi analisado um total de 61 participantes que concluíram o estudo, com 49 7,6 anos de idade, variando de 30 a 59 anos, distribuídos em dois grupos G-CESM ( $n=31)$ e G-controle $(n=30)$, por seis meses. Em geral, houve predominância do sexo feminino $(91,8 \%)$, não brancos $(80,3 \%)$, com companheiro $(68,9 \%)$, escolaridade igual ou inferior a oito anos $(64,5 \%)$, ganhando um salário mínimo ou mais $(63,9 \%)$, não fumante $(80,3 \%)$, sem hábito de beber $(54,1 \%)$ e não praticavam atividade física $(60,7 \%)$. A maioria dos participantes apresentava excesso de peso $(63,9 \%)$, diabetes $(70,5 \%)$ 
e hipertensão (88,5\%). Ambos os grupos tiveram características similares, sem nenhuma diferença significativa entre eles (Tabela 1).

Tabela 1. Características da amostra nos grupos de intervenção e de controle

\begin{tabular}{|c|c|c|}
\hline Características & $\begin{array}{c}\text { G-controle }(\mathrm{n}=30) \\
\mathrm{n}(\%)\end{array}$ & $\begin{array}{c}\text { G-CESM }(n=31) \\
n(\%)\end{array}$ \\
\hline \multicolumn{3}{|l|}{ Sexo } \\
\hline Masculino & $1(3,3)$ & $4(12,9)$ \\
\hline Feminino & $29(96,7)$ & $27(87,1)$ \\
\hline \multicolumn{3}{|l|}{ Cor } \\
\hline Branco & $8(26,7)$ & $4(12,9)$ \\
\hline Não brancos & $22(73,3)$ & $27(87,1)$ \\
\hline \multicolumn{3}{|l|}{ Situação conjugal } \\
\hline Com companheiro & $24(80,0)$ & $18(58,1)$ \\
\hline Sem companheiro & $6(20,0)$ & $13(41,9)$ \\
\hline \multicolumn{3}{|l|}{ Escolaridade } \\
\hline$<8$ anos de estudo & $19(63,3)$ & $20(64,5)$ \\
\hline$>8$ anos de estudo & $11(36,7)$ & $11(35,5)$ \\
\hline \multicolumn{3}{|l|}{ Renda } \\
\hline < 1 salário mínimo & $12(40,0)$ & $9(29,0)$ \\
\hline > 1 salário mínimo & $18(60,0)$ & $22(71,0)$ \\
\hline \multicolumn{3}{|l|}{ Hábito tabágico } \\
\hline Não & $24(80,0)$ & $25(80,6)$ \\
\hline Fuma ou fumou & $6(20,0)$ & $6(19,4)$ \\
\hline \multicolumn{3}{|l|}{ Uso de álcool } \\
\hline Não & $16(53,1)$ & $17(54,8)$ \\
\hline Bebe ou bebeu & $14(45,2)$ & $14(46,7)$ \\
\hline \multicolumn{3}{|l|}{ Prática de atividade física } \\
\hline Não & $18(60,0)$ & $19(61,3)$ \\
\hline Sim & $12(40,0)$ & $12(38,7)$ \\
\hline \multicolumn{3}{|l|}{$\operatorname{IMC}\left(\mathrm{kg} / \mathrm{m}^{2}\right)$} \\
\hline Eutrófico & $3(10,0)$ & $3(9,7)$ \\
\hline Sobrepeso & $21(70,1)$ & $18(58,1)$ \\
\hline Obesidade & $6(20,0)$ & $10(32,2)$ \\
\hline \multicolumn{3}{|l|}{ Diabetes } \\
\hline Não & $7(23,3)$ & $11(35,5)$ \\
\hline Sim & $23(76,7)$ & $20(64,5)$ \\
\hline \multicolumn{3}{|l|}{ Hipertensão } \\
\hline Não & $2(6,7)$ & $5(16,1)$ \\
\hline Sim & $28(93,3)$ & $26(83,9)$ \\
\hline
\end{tabular}

n(\%) - frequência relativa e absoluta; G-controle - grupo controle; G-CESM - grupo cuidar educando na síndrome metabólica; IMC - índice de massa corporal
Em relação ao impacto do programa educativo na qualidade de vida dos participantes, não foi identificada diferença significativa para os domínios na análise entre os grupos (Tabela 2). Entretanto, observou-se que apenas o domínio da capacidade funcional apresentou redução do escore no G-CESM após a intervenção, enquanto isso todos os demais domínios tiveram escores superiores quando comparado com a condição basal do estudo. Além disso, verificou-se diferença estatística para o domínio de dor corporal no grupo G-CESM após o período de intervenção $(\mathrm{p}=0,01)$.

A partir da avaliação do conhecimento sobre SM e dos seus fatores de risco, pode-se notar efeito do programa educativo implementado, uma vez que houve incremento em três das variáveis testadas (hipertensão e diabetes, $\mathrm{p}=0,02$; síndrome metabólica, $\mathrm{p}<0,001$; conhecimento geral, $\mathrm{p}<0,001)$ para o grupo G-CESM, após seis meses de intervenção com sete oficinas temáticas, o que não foi observado no grupo G-controle (Tabela 3). Embora sem diferença estatística entre os grupos.

\section{Discussão}

Este estudo avaliou a efetividade de um programa de intervenção educativa para mudança no conhecimento da SM e dos seus fatores de risco, como também verificou efeitos na melhora da qualidade de vida de adultos com SM, após seis meses de intervenção. O programa de intervenção foi bem recebido pelos participantes, que tiveram acompanhamento completo, representado por um substancial

Tabela 2. Comparações dos domínios de qualidade de vida nos grupos G-controle e G-CESM, nos momentos pré e pós-intervenção

\begin{tabular}{|c|c|c|c|c|c|c|}
\hline \multirow{2}{*}{ Variáveis } & \multicolumn{2}{|c|}{ G-controle $(n=30)$} & \multicolumn{2}{|c|}{ G-CESM $(n=31)$} & \multirow[t]{2}{*}{$p$-value } & \multirow[b]{2}{*}{$p$-value } \\
\hline & Pré & Pós & Pré & Pós & & \\
\hline \multicolumn{7}{|l|}{ SF-36 } \\
\hline Capacidade funcional & $60(45-75)$ & $70(50-90)$ & $75(55-85)$ & $65(55-90)$ & 0,27 & 0,64 \\
\hline Aspectos físicos & $12(0-100)$ & $0(0-100)$ & $75(0-100)$ & $100(25-100)$ & 0,96 & 0,31 \\
\hline Dor corporal & 46 (22-72) & $46,5(22-72)$ & 42 (22-72) & $62(42-100)$ & 0,92 & $0,01^{\#}$ \\
\hline Estado geral de saúde & $52(34-66)$ & 47 (39-67) & $57(30-70)$ & $60(47-72)$ & 0,79 & 0,32 \\
\hline Vitalidade & $52(35-60)$ & $55(45-65)$ & $55(40-65)$ & $65(50-70)$ & 0,06 & 0,06 \\
\hline Aspectos sociais & $94(50-100)$ & $62(38-100)$ & $75(50-100)$ & $88(62-100)$ & 0,25 & 0,20 \\
\hline Aspectos emocionais & $0(0-100)$ & $0(0-100)$ & $100(0-100)$ & $0(0-100)$ & 0,93 & 0,36 \\
\hline Saúde mental & $58(35-68)$ & $58(43-77)$ & 76 (64-92) & $80(64-92)$ & 0,38 & 0,90 \\
\hline
\end{tabular}

Mediana e intervalo interquartil; teste U de Mann-Whitney e teste de Wilcoxon; $\mathrm{p}^{*}$ - significância estatística em relação pré no G-controle; $\mathrm{p}^{*}$ - significância estatística em relação pré no G-CESM; G-controle - grupo controle; G-CESM - grupo cuidar educando na síndrome metabólica; SF-36 - Questionário de Qualidade de Vida SF-36 
Tabela 3. Avaliação de acertos no questionário de conhecimento sobre SM e dos seus fatores de risco nos grupos G-controle e G-CESM, nos momentos pré e pós-intervenção

\begin{tabular}{|c|c|c|c|c|c|}
\hline \multirow{2}{*}{ Variáveis } & \multicolumn{2}{|c|}{ G-controle $(n=30)$} & \multicolumn{2}{|c|}{ G-CESM $(n=31)$} & \multirow{2}{*}{ p-value* } \\
\hline & Pré & Pós & Pré & Pós & \\
\hline \multicolumn{6}{|l|}{ Conhecimento } \\
\hline HAS e diabetes (7 pontos) & $7(6-7)$ & $7(6-7)$ & $6(5-7)$ & $7(6-7)$ & $0,02^{*}$ \\
\hline Tabagismo e álcool(3 pontos) & $1(1-2)$ & $1(1-2)$ & $1(1-1)$ & $1(1-1,5)$ & 0,19 \\
\hline Estresse(3 pontos) & $2(2-2)$ & $2(2-2)$ & $2(2-2)$ & $2(2-2)$ & 0,53 \\
\hline Sedentarismo(3 pontos) & $1(1-2)$ & $1(1-2)$ & $1(1-2)$ & $1(1-1)$ & 0,06 \\
\hline Medicamento(2 pontos) & $1(1-1)$ & $1(1-1)$ & $1(1-1)$ & $1(1-1)$ & 0,41 \\
\hline Alimentação(9 pontos) & $7(6-7)$ & $7(6-7)$ & $7(6-7)$ & $7(7-7)$ & 0,17 \\
\hline Síndrome Metabólica(9 pontos) & $0(0-1)$ & $0(0-0)$ & $0(0-1)$ & $8(8,5-9,0)$ & $<0,01^{*}$ \\
\hline Conhecimento geral(36 pontos) & $20,5(19-22)$ & $20,5(20-23)$ & $20(18,5-21)$ & $28(26-29)$ & $<0,01^{*}$ \\
\hline
\end{tabular}

Mediana e intervalo interquartil; teste U de Mann-Whitney e teste de Wilcoxon; $\mathrm{p}^{\star}$ - significância estatística em relação pré no mesmo grupo; G-controle - grupo controle; G-CESM - grupo cuidar educando na síndrome metabólica; HAS - hipertensão

percentual de adesão (76,3\%). Os participantes, em sua maioria, eram obesos ou sobrepesados, diabéticos, hipertensos e não praticavam atividade física.

As oficinas do programa tiveram abordagem multidisciplinar de promoção da saúde para adultos com SM, incluindo a orientação e liderança de enfermagem como uma novidade. Os resultados sugerem que o grupo G-CESM demostrou melhora para a maioria dos domínios de qualidade de vida dos participantes, com significativo aumento no escore de dor corporal. Outra contribuição importante deste estudo foi ter demonstrado efeitos no conhecimento sobre hipertensão, diabetes, SM e do conhecimento geral dos fatores de risco da SM em adultos, com baixo nível de escolaridade ( $<8$ anos), após o final da intervenção.

Ainda em relação ao conhecimento, o aumento de pontos significativos para SM no grupo G-CESM é um achado que merece atenção, pois mostra a importância da realização de oficinas em grupo como tecnologia de promoção da saúde no cuidado e rastreio de adultos com SM atendidos pelo sistema público de saúde, confirmando em parte a hipótese investigada.

Estudos que investigaram efeitos de programas de promoção da saúde observaram melhoras dos domínios de qualidade de vida em adultos com SM. ${ }^{(5-8,17)}$ Por exemplo, uma intervenção no estilo de vida de mulheres com SM ( $n=52 ; 62,7 \pm 9,0$ anos), em três centros comunitários de saúde, mostrou melhora significativa para aspectos físicos, estado geral de saúde, vitalidade e saúde mental da qualidade de vida, aos seis meses e se manteve até 12 meses depois da intervenção. ${ }^{(5)}$ Já o escore de dor corporal no grupo intervenção aumentou após seis meses de intervenção, em comparação a uma diminuição do escore no grupo controle no mesmo período, embora sem diferença significativa. ${ }^{(5)}$

No estudo ${ }^{(17)}$ ( $\mathrm{n}=58 ; 48,6 \pm 5,8$ anos) também foi verificado aumento significativo no escore de dor corporal, após receber intervenção de terapia cognitiva comportamental, com discussão em formato de grupo sobre hábitos alimentares, realização de exercícios, controle do estresse e respiração. Outro estudo ( $n=160 ; 44,2 \pm 10,0$ anos) pontuou melhora no domínio de dor corporal no grupo intervenção aos três e seis meses após aplicação de um programa de website para promoção da saúde, que produziu resultados significativos na adoção de uma alimentação saudável e hábitos de atividade física. ${ }^{(18)}$ Esses assuntos são consistentes com os abordados no programa investigado neste estudo. Acredita-se que as orientaçóes, especialmente em grupo, podem ter levado os participantes a adotarem comportamentos recomendados de prevenção e cuidados durante o tratamento da SM, o que pode ter ajudado a aumentar os escores da maioria dos domínios da qualidade de vida e causar melhora significativa da dor corporal, que também foi um dos assuntos discutido nas oficinas.

Há evidências de que programas de intervenção com o objetivo de estimular a prática de atividade física e hábitos alimentares tiveram melhora dos domínios de qualidade de vida SM..$^{(10,19)}$ Os resultados dos programas se tornam ainda mais relevantes por mostrarem diminuição do peso corporal, circunferência abdominal, IMC, da pressão arterial, HDL-c e triglicerídeos, ${ }^{(5,6,8,10,17,19)}$ pois são considerados in- 
dicadores que podem comprometer qualidade de vida e aumentar o risco de desenvolver a SM. ${ }^{(7)}$

A melhora dos domínios da qualidade de vida no grupo G-CESM, sugere uma mudança de comportamentos no estilo de vida para adoção de hábitos mais saudáveis, a partir de uma abordagem multidisciplinar com envolvimento dos participantes. Logo, o programa investigado fornece uma alternativa para melhorar a dor corporal da qualidade de vida em adultos com SM, usuários de um centro de saúde. É reconhecido que os centros de saúde no âmbito da atenção primária são ambientes ideais para desenvolver intervenções no estilo de vida, como a testada neste estudo, porque favorece uma importante combinação entre cuidado à saúde e apoio social. ${ }^{(20,21)}$

Manter o comportamento saudável ao longo de dois anos foi associado a maiores chances de não desenvolver SM. As intervençóes em grupo produzem fatores essenciais para adesão e manutenção de um estilo de vida saudável, por possibilitar a comunicação, apoio emocional, motivação e tomada de decisão compartilhada entre os participantes e profissionais de saúde. Depois que o participante adquire o conhecimento sobre a SM, permitirá a ele gerenciar melhor seus hábitos e manter práticas de comportamentos saudáveis no seu cotidiano. É nesse momento que o acompanhamento contínuo pelos profissionais de saúde pode garantir a continuidade da promoçáo do bem-estar, conforme demonstrado por pesquisas anteriores. ${ }^{(6,22-24)}$

A melhora do conhecimento é relevante, pois as características dos participastes contribuem para o desconhecimento da SM. Como visto anteriormente, que o baixo nível de escolaridade e, principalmente, por se conhecer que pessoas diabéticas apresentam comprometimento da função cognitiva e capacidade física, que se agrava com o avançar da idade (>55 anos). ${ }^{(25)}$ Além disso, indivíduos com sobrepeso e obesidade demostraram desconhecimento da SM e seus fatores de risco. ${ }^{(26,27)}$ Portanto, indica a necessidade de intervenção de educação em saúde para esse grupo em risco.

Outro estudo transversal, ${ }^{(3)}$ também identificou baixo nível de conhecimento da SM em adultos ( $\mathrm{n}=$ $113 ; 58,5 \pm 10,1$ anos), com fatores de risco cardio- metabólicos. E demonstrou resultados interessantes, verificou-se que as variáveis de maior tempo de escolaridade (entre 9 a 12 anos ou > 12 anos), história pregressa de dislipidemia e nível normal de HDL-c são características preditoras do conhecimento sobre SM, muito provavelmente, devido a relação de causa-efeito entre o aumento do conhecimento da SM e melhor gerenciamento do cuidado de si mesmo. ${ }^{(3)}$

Portanto, a constatação do desconhecimento da SM na linha de base dos dois grupos concordam com achados de estudos transversais anteriores. ${ }^{(2,21)}$ Satisfatoriamente, conseguiu-se melhorar o nível de conhecimento sobre SM e dos seus fatores de risco após seis meses de intervenção de um programa educativo de promoção da saúde. Embora atualmente haja poucas evidências de intervençôes educativas no conhecimento da SM, houve um significativo aumento do conhecimento para outras doenças crônicas, como hipertensão e diabetes. Com base em nossas descobertas, a implementação de programas de promoção da saúde com enfoque para SM parece melhorar o comportamento dos adultos, conforme recomendações de prevenção e tratamento da SM.

Os resultados deste estudo mostram que um programa educativo de promoção da saúde melhora significativamente a dor corporal da qualidade de vida de adultos com SM. Embora não tenha havido significância estatística, o escore da maioria dos domínios da qualidade de vida no G-CESM aumentou ao longo do estudo, o que não foi observado no grupo controle. Ainda, contribuiu para aumentar o conhecimento geral dos fatores de risco da SM, com incremento de conhecimento sobre hipertensão, diabetes e da própria $\mathrm{SM}$ em relação à linha de base para final da intervenção. Essas alterações podem fornecer benefícios clínicos para adultos com SM em centros comunitários de saúde.

Apesar dos resultados satisfatórios dessa intervenção, este estudo tem algumas limitaçóes. Utilizou-se uma amostra por conveniência, devido à especificidade dos critérios de elegibilidade. Além disso, a participação voluntária restrita a adultos de um único centro de saúde pode ter contribuído para o pequeno tamanho amostral. Essas condições podem limitar a generalização dos resultados. Outro fato, é que todos os adultos pertencem à mesma 
comunidade e, nesse caso, o cegamento dos participantes não seria possível. Apesar disso, os grupos ainda apresentam diferenças importantes em seus resultados. $\mathrm{O}$ estudo investigou os efeitos em curto prazo (seis meses). Estudos randomizado de longo prazo que incluam mais centros de saúde são necessárias para verificar se essas alteraçóes na qualidade de vida e no conhecimento da SM são mantidas. Contudo, nosso programa forneceu acompanhamento de enfermagem com avaliação antropométri$\mathrm{ca}$, de fatores de risco cardiometabólico e conhecimentos específicos de promoção da saúde para SM, orientado por especialistas durante seis meses.

Este estudo sugere que um programa educativo liderado pela enfermagem promova à saúde por meio de mudanças do estilo de vida, capazes de melhorar a qualidade de vida, especialmente a dor corporal e o conhecimento dos fatores de risco para SM, em adultos com SM. Esse resultado é clinicamente relevante, pois foi alcançado sem prescrição de nenhum tipo de dieta restritiva ou atividade física de alta intensidade. Considera-se que esse programa é economicamente viável e não acrescenta carga de trabalho elevada aos profissionais de saúde dos centros de atençáo primária. Certamente, esses fatores foram determinantes para atingir resultados satisfatórios do programa investigado.

\section{Conclusão}

A intervenção de um programa educativo de promoção da saúde em grupo, ao longo de seis meses, melhorou a avaliação da qualidade de vida com efeito significativo para o escore de dor corporal em adultos com SM. Como também houve melhora no conhecimento geral da SM e dos seus fatores de risco. Os resultados confirmaram que o programa investigado pode ser uma estratégia eficiente e baixo custo para os centros da atenção primária de saúde.

\section{Colaborações}

Santos ISC, Araújo WA, Damaceno TO, Souza AS, Boery RNSO e Fernandes JD contribuíram com a concepção do estudo, análise e interpretação dos dados, redação do artigo, revisão crítica relevante do conteúdo intelectual e aprovação da versão final a ser publicada.

\section{Referências}

1. Félix ND, Nóbrega MM. Metabolic syndrome: conceptual analysis in the nursing context. Rev Lat Am Enfermagem. 2019;27:e3154. Review.

2. Grundy SM, Cleeman JI, Daniels SR, Donato KA, Eckel RH, Franklin BA, Gordon DJ, Krauss RM, Savage PJ, Smith SC Jr, Spertus JA, Costa F; American Heart Association; National Heart, Lung, and Blood Institute. Diagnosis and management of the metabolic syndrome: an American Heart Association/National Heart, Lung, and Blood Institute Scientific Statement. Circulation. 2005;112(17):2735-52. Erratum in: Circulation. 2005;112(17):e297. Erratum in: Circulation. 2005;112(17):e298. Review.

3. Wang Q, Chair SY, Wong EM, Taylor-Piliae RE, Qiu XCH, Li XM. Metabolic syndrome knowledge among adults with cardiometabolic risk factors: a cross-sectional study. Int J Environ Res Public Health. 2019;16(1):159.

4. Oliveira LV, Santos BN, Machado ÍE, Malta DC, Velasquez-Melendez G, Felisbino-Mendes MS. Prevalence of the metabolic syndrome and its components in the Brazilian adult population. Cien Saude Colet. 2020;25(11):4269-80.

5. Oh EG, Bang SY, Hyun SS, Kim SH, Chu SH, Jeon JY, et al. Effects of a 6-month lifestyle modification intervention on the cardiometabolic risk factors and health-related qualities of life in women with metabolic syndrome. Metabolism. 2010;59(7):1035-43.

6. Wang $Q$, Chair SY, Wong EM. The effects of a lifestyle intervention program on physical outcomes, depression, and quality of life in adults with metabolic syndrome: a randomized clinical trial. Int $\mathrm{J}$ Cardiol. 2017;230:461-7.

7. Saboya PP, Bodanese LC, Zimmermann PR, Gustavo AD, Macagnan $F E$, Feoli AP, et al. Lifestyle intervention on metabolic syndrome and its impact on quality of life: a randomized controlled trial. Arq Bras Cardiol. 2017;108(1):60-9.

8. Li C, Sadraie B, Steckhan N, Kessler C, Stange R, Jeitler M, et al. Effects of a one-week fasting therapy in patients with type-2 diabetes mellitus and metabolic syndrome - a randomized controlled explorative study. Exp Clin Endocrinol Diabetes. 2017;125(9):618-24.

9. Tran VD, James AP, Lee AH, Jancey J, Howat PA, Thi Phuong Mai L. Effectiveness of a community-based physical activity and nutrition behavior intervention on features of the metabolic syndrome: a cluster-randomized controlled trial. Metab Syndr Relat Disord. 2017;15(2):63-71.

10. Frere NO, Soliman SS, Foda MA, Eyada TK, Saad NE. Effect of patient education on metabolic syndrome components among females in Zagazig University outpatient clinics, Egypt: an intervention study. Diabetes Metab Syndr. 2019;13(3):1897-900.

11. Freire P. Pedagogia da autonomia: saberes necessários à prática educativa. São Paulo: Paz e Terra; 2011.

12. I Diretriz Brasileira de Diagnóstico e Tratamento da Síndrome Metabólica [Editorial]. Arq Bras Cardiol. 2005;84(Suppl 1):3-28.

13. Malachias MV, Plavnik FL, Machado CA, Malta D, Scala LC, Fuchs S. $7^{\text {a }}$ Diretriz Brasileira de Hipertensão Arterial: Capítulo 1 - Conceituação, Epidemiologia e Prevenção Primária. Arq Bras Cardiol. 2016;107(3 Suppl 3):1-6. 
14. Sociedade Brasileira de Diabetes. Diretrizes da Sociedade Brasileira de Diabetes 2017-2018. São Paulo: Clannad; 2017 [citado 2021 Abr 11]. Disponível em: https://www.diabetes.org.br/profissionais/ images/2017/diretrizes/diretrizes-sbd-2017-2018.pdf

15. Associação Brasileira para o Estudo da Obesidade e da Síndrome Metabólica - ABESO. Diretrizes brasileiras de obesidade 2016. 4a ed. São Paulo: Associação Brasileira para o Estudo da Obesidade e da Síndrome Metabólica; 2016 [citado 2021 Abr 11]. Disponível em: $\quad$ https://abeso.org.br/wp-content/uploads/2019/12/DiretrizesDownload-Diretrizes-Brasileiras-de-Obesidade-2016.pdf

16. Ciconelli RM, Ferraz MB, Santos W, Meinão I, Quaresma MRI. Tradução para o português e validação do questionário genérico de avaliação de qualidade de vida SF-36 (Brasil SF-36). Rev Bras Reumatol. 1999;39(3):143-50.

17. Zhang Y, Mei S, Yang R, Chen L, Gao H, Li L. Effects of lifestyle intervention using patient-centered cognitive behavioral therapy among patients with cardio-metabolic syndrome: a randomized, controlled trial. BMC Cardiovasc Disord. 2016;16(1):227.

18. Jahangiry L, Montazeri A, Najafi M, Yaseri M, Farhangi MA. An interactive web-based intervention on nutritional status, physical activity and healthrelated quality of life in patient with metabolic syndrome: a randomizedcontrolled trial (The Red Ruby Study). Nutr Diabetes. 2017;7(1):e240.

19. Watanabe M, Yokotsuka M, Yamaoka K, Adachi M, Nemoto A, Tango T. Effects of a lifestyle modification programme to reduce the number of risk factors for metabolic syndrome: a randomised controlled trial. Public Health Nutr. 2017;20(1):142-53.

20. Rosas LG, Lv N, Xiao L, Lewis MA, Zavella P, Kramer MK, et al. Evaluation of a culturally-adapted lifestyle intervention to treat elevated cardiometabolic risk of Latino adults in primary care (Vida Sana): a randomized controlled trial. Contemp Clin Trials. 2016;48:30-40.

21. Smith SM, Wallace E, O'Dowd T, Fortin M. Interventions for improving outcomes in patients with multimorbidity in primary care and community settings. Cochrane Database Syst Rev. 2016;3(3):CD006560. Update in: Cochrane Database Syst Rev. 2021;1:CD006560.

22. VanWormer JJ, Boucher JL, Sidebottom AC, Sillah A, Knickelbine T. Lifestyle changes and prevention of metabolic syndrome in the Heart of New Ulm Project. Prev Med Rep. 2017;6:242-5.

23. Schalock RL, Verdugo MA, Gomez LE, Reinders HS. Moving us toward a theory of individual quality of life. Am J Intellect Dev Disabil. 2016;121(1):1-12.

24. Thom $G$, Lean $M$. Is there an optimal diet for weight management and metabolic health? Gastroenterology. 2017;152(7):1739-51. Review.

25. Mallorquí-Bagué N, Lozano-Madrid M, Toledo E, Corella D, SalasSalvadó J, Cuenca-Royo A, et al. Type 2 diabetes and cognitive impairment in an older population with overweight or obesity and metabolic syndrome: baseline cross-sectional analysis of the PREDIMED-plus study. Sci Rep. 2018;8(1):16128.

26. Salazar NA, Oviedo LM, Samamé LT, Tell NM. Conocimiento sobre síndrome metabólico en pacientes con sobrepeso u obesidad de un hospital de alta complejidad de lambayeque, 2016. Rev Exp Med. 2018;4(2):56-60.

27. Peiris CL, Taylor NF, Hull S, Anderson A, Belski R, Fourlanos S, et al. A group lifestyle intervention program is associated with reduced emergency department presentations for people with metabolic syndrome: a retrospective case-control study. Metab Syndr Relat Disord. 2018;16(2):110-6. 